\title{
Necrotizing Enterocolitis, Gut Microbiota, and Brain Development: Role of the Brain-Gut Axis
}

\author{
Hendrik J. Niemarkt ${ }^{\mathrm{a}} \quad$ Tim G. De Meij $^{\mathrm{b}}$ Christ-jan van Ganzewinkel ${ }^{\mathrm{a}}$ \\ Nanne K.H. de Boer ${ }^{c}$ Peter Andriessen ${ }^{a}$ Matthias C. Hütten ${ }^{d}$ Boris W. Kramer ${ }^{d}$

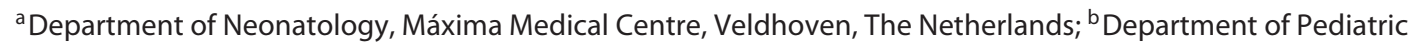 \\ Gastroenterology, Amsterdam University Medical Centre, Amsterdam, The Netherlands; ' Department of \\ Gastroenterology and Hepatology, AG\&M Research Institute, Amsterdam University Medical Centre, Vrije \\ Universiteit Amsterdam, Amsterdam, The Netherlands; ${ }^{\mathrm{d}}$ Department of Pediatrics, Maastricht University Medical \\ Centre, Maastricht, The Netherlands
}

\section{Keywords}

Necrotizing enterocolitis · Brain development · Brain-gut axis . Preterm infants

\begin{abstract}
Necrotizing enterocolitis (NEC) is a relatively common disease in very-low-birth-weight infants and is associated with high mortality and morbidity. In survivors, neurodevelopmental impairment is frequently seen. The exact etiology remains largely to be elucidated, but microbiota are considered to play a major role in the development of NEC. Furthermore, emerging evidence exists that the microbiota is also of importance in brain function and development. Therefore, microbiota characterization has not only potential as a diagnostic or even preventive tool to predict NEC, but may also serve as a biomarker to monitor and possibly even as a target to manipulate brain development. Analysis of fecal volatile organic compounds, which shape the volatile metabolome and reflect microbiota function and host interaction, has been shown to be of interest in the diagnosis of NEC
\end{abstract}

and late-onset sepsis. In this review, we discuss evidence of the role of the complex interplay between microbiota, NEC, and brain development, including the brain-gut axis in preterm infants.

(C) 2019 The Author(s)

Published by S. Karger AG, Basel

\section{Introduction}

Necrotizing enterocolitis (NEC) is the commonest severe gastrointestinal disease in very-low-birth-weight infants, with reported incidence rates of between 3 and $15 \%$. NEC is associated with a mortality rate of between 15 and $30 \%[1,2]$. Survivors of NEC often have life-long consequences, such as short-bowel syndrome and impaired neurological outcome [3]. The pathophysiology is not clear but is considered to be multifactorial. Immaturity of the gut, hypoxia-ischemia, enteral (namely formula) feeding, and microbial dysbiosis play a role in inducing an inflammatory response in the gut. This inflammatory cascade leads, especially at the clinical onset of the dis- 
ease, to non-specific symptoms which can resemble sepsis, hampering a swift clinical diagnosis and thus appropriate therapy. As the etiology of NEC is not clear, early intervention aimed at targeted, scientific-based manipulation of the pathophysiological pathways is not feasible. The clinical picture of NEC may represent different disease pathways with the final outcome of intestinal necrosis [4-6]. Little progress has been made in the diagnosis and treatment of NEC in recent decades, partly due to the unclear etiology and definition.

Although no single pathogen has been identified to cause NEC, there has been an increasing understanding of the relation between overall gut microbiota, compositional alterations referred to as dysbiosis, and NEC $[5,7]$. The recognition of NEC-specific dysbiosis and/or the alteration of the microbiota may allow for a timely diagnosis, targeted treatment, and possibly even prevention or treatment of NEC. Furthermore, the inflammatory cascade induced by microbiota in NEC is considered not to be limited to the gut but may also influence the brain via the so-called bidirectional gut-brain axis [8].

Technological advances and the availability of new molecular and analytic techniques have provided more knowledge on the human microbiota, metabolic activity and the role microbiota play in health and disease. The microbiota in neonates is of interest as it goes through several developmental stages and is involved with the development of the immune system and brain. Neurocognitive development is often impaired in NEC and microbiota are supposed play an important role in developmental processes in neonates. It is of interest to study microbiota with respect to their gastrointestinal consequences and also their influence on brain development in NEC.

Fig. 1. Schematic overview of the role of microbiota in brain development and functioning and in NEC, and summary of the effects of the gut microbiota on brain, gut motility and inflammation. The gut microbiota are highly metabolically active. Bacterial metabolites have multiple effects on the gut and brain development, and function by different pathways, including the circulation and vagal nerve (top panel). In NEC, LPS-containing microbes can inflame the brain by TLR4-induced cytokine release. SCFAs, produced by microbial processes, are of importance in maintaining gut barrier functioning, and may have a direct effect on brain functioning. Recently, it was recognized that the gut microbiota produced metabolites that signal to colonic enterochromaffin cells (ECs). ECs increase serotonin (5-HT) biosynthesis, resulting in the secretion of 5-HT into the lumen and basolaterally (lower left panel). This increases the stimulation of myenteric neurons, resulting in gut motility. Besides, microbes can play a direct role in the production different neurotransmitters, such as GABA and 5-HT. In a symbi-
In this review, we discuss the role of microbiota in the etiology of NEC and how signs of gut dysbiosis may help in the early diagnosis of NEC. In addition, we will discuss the neurocognitive consequences of NEC and how the microbiota may mediate these in NEC survivors.

\section{Microbiota and the Gut-Brain Axis}

The gut-brain axis is a communication system that integrates neural, hormonal, and immunological signaling between the gut and the brain, and provides the intestinal microbiota and its metabolites with a potential route to influence brain function and development (Fig. 1). However, the exact mechanisms by which the gut microbiota communicate with the brain have not been elucidated yet [9].

Most compelling evidence for the existence of a gutbrain axis is derived from experimental studies in germfree mice (gnotobiotic mice). For example, germ-free mice showed exaggerated stress-anxiety behavior with increased cortisone and adreno-cortisone levels in response to mild stress, compared with specific-pathogen-free mice [10]. Interestingly, when germ-free mice were colonized with the fecal material of the specific-pathogen-free mice, their behavior normalized. In the germ-free mice, decreased concentrations of neurotrophic factor were found, which are associated with brain plasticity [11]. Diaz Heijtz [12] reported changes of synaptophysin and PDS-95, which are proteins involved in synaptogenesis, after changing the gut microbiota in mice. Germ-free mice also showed different levels of neurotransmitters, such as noradrenalin, dopamine, and serotonin, compared to colonized mice.

otic condition, the gut microbiota is mainly comprised of obligate anaerobic bacteria. The gut microbiota regulate hemostasis of the intestinal epithelial cells (lower right hand panels). In a normal, symbiotic situation the intestinal lumen is kept hypoxic by betaoxidation, maintaining the growth of anaerobic bacteria partially by the activity of nitric oxide synthase (NOS) 2 . The SCFA butyrate is a key microbial metabolite since it is detected by the intracellular peroxisome proliferator-activated receptor- $\gamma($ PPAR- $\gamma)$, which activates beta-oxidation in macrophages. After treatment with antibiotics or other environmental factors which may provoke the dominance of pathogenic bacteria (dysbiosis), the metabolism changes to glycolysis, which favors proliferation of pathogenic bacteria. The gut immune system is in balance/not activated in a symbiotic situation. However, dysbiosis (upper and lower panels) results in a systemic inflammatory response that can be overwhelming, resulting in NEC.

(For figure see next page.) 


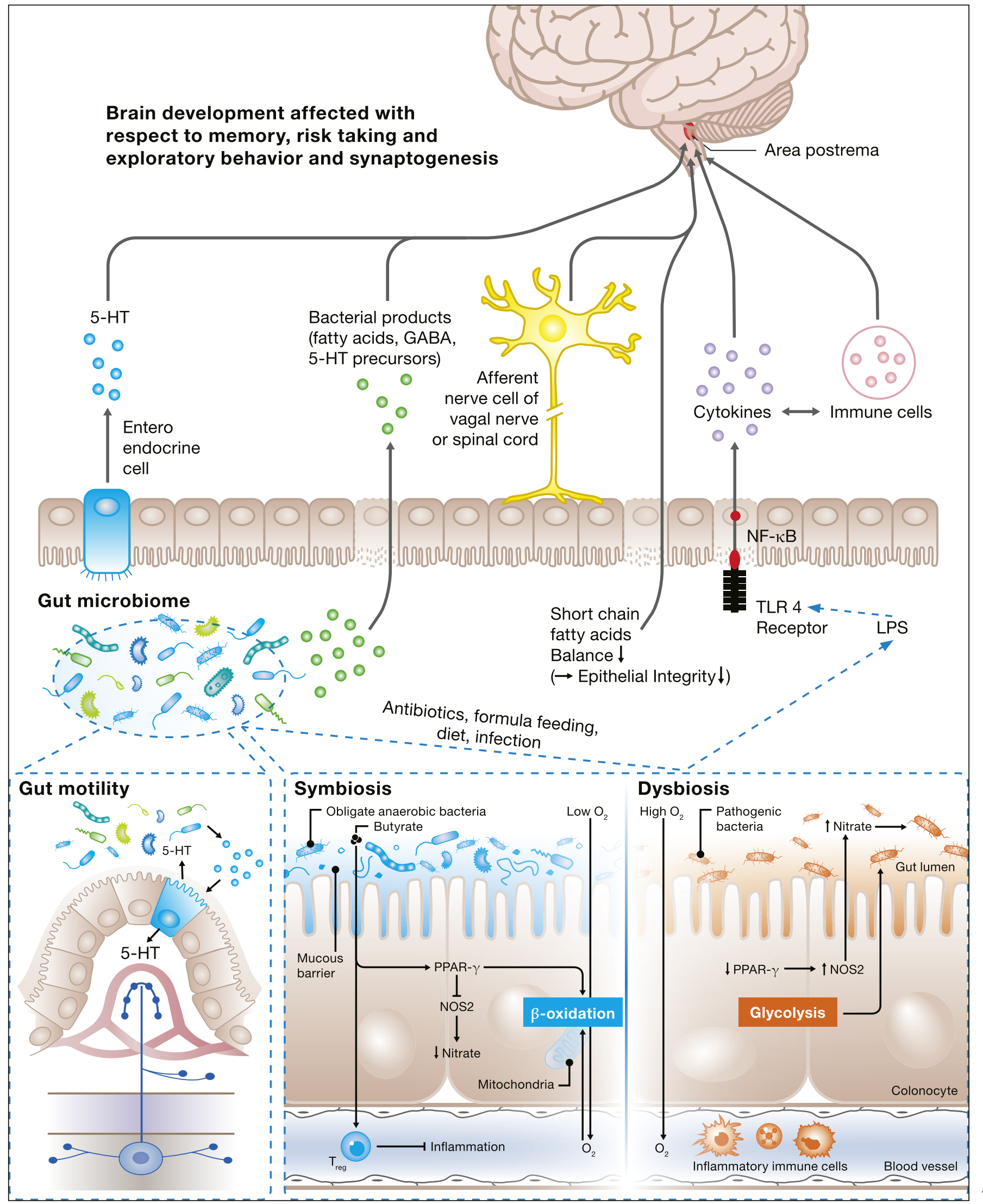

NEC, Gut Microbiota, and Brain

Neonatology 2019;115:423-431

DOI: $10.1159 / 000497420$ 
Another example of the existence of a gut-brain axis was demonstrated by Lyte et al. [13] who showed behavioral changes in mice after infection with Campylobacter jejuni. Following infection, these animals displayed a significant increase in anxiety-like behavior. In line with this observation, Goehler et al. [14] showed that C. jejuni activated vagal ascending pathways, which are specific pathways connected to anxiety-like behavior. Furthermore, mice administered with the probiotic strain Lactobacillus rhamnosus expressed changes in brain neurotransmitter concentrations and showed a reduction in anxiety-like behavior. This effect was not seen in vagotomized mice, indicating that the vagal nerve is an important pathway for communication between intestinal microbiota, gut, and brain [11].

Microbiota can produce a variety of neurotransmitter compounds: serotonin, dopamine, noradrenalin, and acetylcholine, and gamma-aminobutyric acid (GABA) can be produced by several genera of bacteria [15-17]. In experiments with rats, administration of the probiotic strain $B i$ fidobacterium longum resulted in normalization of depression-like behavior after separation from their mother. This was accompanied by changes in noradrenalin and corticotrophin levels in the brain. B. longum administration in rats was also associated with changes in cortical levels of the neurotransmitters dopamine and serotonin [18]. In humans, the neurotransmitter GABA, which is related to anxiety, has recently been shown to be produced by commensal lactobacilli and bifidobacteria [9].

Short-chain fatty acids (SCFAs), which are produced by microbial-initiated fermentation processes in the gut, have been suggested to promote the integrity of the gut barrier and the blood-brain barrier [12]. SCFAs have also been shown to influence behavior in animals and humans. For example, high fecal concentrations of propionic acid correlate with anxiety in patients with irritable bowel disease [9]. In addition, carbohydrate malabsorption, which leads to increased substrate availability for fermentation, is associated with depression in human females [19]. Furthermore, alterations in SCFA levels have been linked to Alzheimer, Parkinson, and Huntington diseases [16].

Finally, microbiota may alter brain development by mediating the immune system (Fig. 1). Changes in microbial composition induced by environmental factors, including diet and probiotics or antibiotics, may alter cytokine profiles mediated by the activation of Toll like receptors (TLRs) [20]. For example, B. longum supplementation in rats induced secretion of IL- 6 by peripheral blood cells and improved depression-like behavior [18].
These results suggest the ability of the gut microbiota to modulate brain development and behavior and propose a critical window for intestinal microbes to influence developmental programming of long-lasting brain function [21]. With respect to this concept, the gut microbiota constitutes an interesting target for potential neuroprotective strategies in preterm infants.

\section{Microbiota and NEC}

The role of bacterial exposure in the development of NEC cannot be underestimated. Some $90 \%$ of infants who develop NEC were fed prior to the onset of disease and feeding is an important factor in the composition of the microbiota. Experimental models have shown the pivotal role of bacteria in its pathogenesis as germ-free animals could not develop NEC $[22,23]$. Although no specific pathogen has been found to meet the Koch postulates in the development of NEC, Claud and Walker [24] introduced the concept that NEC is associated with inappropriate colonization of the preterm gut.

Classically, the fetal environment was considered to be sterile. However, recent studies suggest that colonization of the gastrointestinal tract may already have begun in utero [25]. Commensal bacteria colonizing the gut are usually derived from maternal colonic and vaginal flora [26], although recent studies identified a unique microbiome in the placenta, which might play a role as a possible source of neonatal commensal microbiota [27]. These commensal bacteria maintain intestinal homeostasis, protect the gut from injury, and have an immune-regulatory function $[28,29]$, while conversely the human intestine adapts physiologically to the increased postnatal microbial colonization [29].

There are several hypotheses on how commensal bacteria may promote NEC in preterm infants. Some authors emphasize the role of an abnormal microbial colonization when infants are born preterm. A recent study showed that colonization of the gut in very-lowbirth-weight infants took place in an orderly process, mainly from an abundance of bacilli via Gammaproteobacteria to Clostridia [30]. Interestingly, in these infants, antibiotics, feeding, or mode of delivery caused abrupt shifts in microbiota but did not change the "predestined" sequence of colonization. Conversely, in term infants the mode of delivery, type of infant feeding, gestational age, infant hospitalization, and antibiotic use highly influenced gut microbiota at 1 month of age [31]. 
In a meta-analysis by Pammi et al. [32], NEC was preceded by an altered intestinal microbiota. Overall, this dysbiosis was characterized by increased relative abundances of Proteobacteria and decreased relative abundances of Firmicutes and Bacteroidetes. No differences in alpha-diversity (richness and evenness of different microbes within samples) and beta-diversity (richness and evenness of microbes between samples) was observed comparing infants with NEC and controls [32]. However, some studies demonstrated that the specific microbiota profile was dependent on the age of disease onset. Infants developing early NEC (within 10 days of life) demonstrated a dominance of Firmicutes, largely attributable to increased abundances of bacilli class members [33-35]. Different bacteria of the Clostridia class, also within the phylum of Firmicutes, were also associated with early-onset NEC. Concurrent with this increase in Firmicutes, a decrease in Gammaproteobacteria was observed in these early-onset cases. Late-onset NEC (after 10 days of life) was associated with an increase in Gammaproteobacteria with an accompanying decrease in Firmicutes (especially Negativicutes) [35, 36].

Antibiotics have pivotal effects (Fig. 1). Infants who received antibiotics showed increased relative abundances of Proteobacteria and decreased relative abundances of Firmicutes and Actinobacteria [32]. This may explain the increased risk of NEC after antibiotic administration in preterm infants. The mode of delivery leads to differences in microbiota, but these were not related to NEC. The relative abundance of Firmicutes was higher after Caesarean section, and of Bacteroides was higher after vaginal delivery. Infants who were fed formula showed a higher abundance of Firmicutes, and infants who were fed breast milk showed higher relative abundances of Proteobacteria, but this was not related to the development of NEC [32]. Another study on enteral feeding type showed that preterm infants who received breast milk showed less interindividual variance in microbiota compared to formula-fed infants [37]. This suggests that the microbiota of formula-fed infants may be more susceptible to environmental factors. The fact that preterm infants who were fed exclusively breast milk have a lower risk of NEC may be based on the fact that, besides the fact that breast milk contains specific macronutrients, polyunsaturated fatty acids, lactoferrin, immune cells, and immunoglobulins, it also contains microbes that appear to be personalized for each mother-infant pair [7].

Dysbiosis may induce inflammation in the preterm gut causing the clinical picture of NEC (Fig. 1). In the model for NEC proposed by Hackam et al. [38] and Neu

NEC, Gut Microbiota, and Brain

Development: Role of the Brain-Gut Axis and Pammi [39], an excessive TLR4 response to lipopolysaccharides (LPS) of gut bacteria (most often Gammaproteobacteria) takes place. Activation of these TLRs results in signaling cascades that induce nuclear translocation of nuclear factor kappa- $\beta$, a transcription factor that induces transcription of various pro-inflammatory and antiinflammatory cytokines and chemokines. Activation of IL-8 attracts immune cells (neutrophils) to trigger areas of the intestine where they undergo phagocytosis and other inflammatory responses that cause vasoconstriction, ischemia, and injury to the intestine (Fig. 1) [7, 40].

Two empiric findings underline this pivotal importance of TLR4 in NEC, the expression of which is typically higher in preterm infants. First, in TLR mutant or knockout animals, NEC is not inducible. Second, probiotic bacteria that activate TLR9, which in turn limits the degree of TLR4 signaling, are associated with reduced NEC in clinical studies [38, 41, 42].

SCFAs, such as butyrate, propionate, and acetate, are produced in metabolic processes by gut bacteria and are known to play a role in barrier function of the intestinal epithelial cells (Fig. 1). Besides, the beta-oxidation of butyrate leads to a low oxygen and nitrate environment in the gut, which helps to prevent dysbiosis [43]. Dysbiosis may disturb the balance in SCFA content in the gut [7]; however, the exact mechanisms have not been elucidated and may be contradictory since both high and low intestinal SCFA levels are associated with NEC (Fig. 1) [39, 44].

Gut microbial-derived metabolites also affect peristalsis. Microbiota products such as bile acids and SCFA affect the production of serotonin, which in turn regulates gut motility (Fig. 1) [45]. Interestingly, the number of spontaneous gut passages has been related to NEC development, suggesting that microbiota have an effect on both gut function and subsequent disease.

\section{Neurodevelopment in Survivors of NEC}

\section{Neurocognitive Outcome}

The relationship between NEC and neurocognitive impairments has been demonstrated by different investigators. Two large systematic reviews confirmed the association between NEC and neurocognitive outcome. Schulzke et al. [46] analyzed 4,239 infants ( 427 with NEC) from 11 studies and calculated an odds ratio (OR) of 1.82 (95\% CI 1.46-2.27) for neurodevelopmental delay in infants with NEC. They also found that infants with surgically treated NEC had a higher risk of neurodevelopmen- 
tal delay compared to infants with medically treated NEC (OR 1.99; 95\% CI 1.25-3.14). Rees et al. [47] analyzed the data from 7,843 preterm infants (821 with NEC) and found a higher risk of neurodevelopmental impairment in infants with NEC (OR 1.58; 95\% CI 1.25-1.99) compared to controls, and a higher risk of impairment in infants with surgical NEC compared to medical NEC (OR 2.34 ; 95\% CI 1.51-3.60).

The effect of NEC on neurocognitive outcome may partly be mediated by comorbidity. Most importantly, sepsis is often accompanied by NEC. Sepsis itself is correlated with an adverse neurocognitive outcome [48]. Indeed, in their extremely low gestational age newborn cohort, Martin et al. [49] demonstrated that risks of neurodevelopmental impairment were higher in infants with surgically treated NEC and concurrent sepsis. NEC is also associated with other complications of prematurity, including mechanical ventilation, bronchopulmonary dysplasia, intraventricular hemorrhage, and retinopathy of prematurity, which affect neurocognitive outcome [3].

\section{Proposed Mechanism of the Relation between NEC and Neurocognitive Outcome}

During NICU admission, the brain of the preterm infant goes through a critical phase of development. This period is marked by rapid brain development including neurogenesis, neuronal migration, maturation apoptosis, and synaptogenesis. Different factors may disrupt these processes, such as malnutrition, hypoxia-ischemia, and cytotoxic mediators that originate from inflammatory processes. It is conceivable that NEC as a disease that takes place during this critical and vulnerable stage of brain development plays a role in neurodevelopmental disorders. The association between NEC and neurodevelopment is most likely multifactorial, with different insults accompanying the intestinal manifestations of NEC. The specific type, severity, and duration of these insults will determine the specific locations of brain injury and outcomes $[3,21]$.

\section{NEC and Chronic Pain}

The systemic and local inflammation, bowel extension, and ischemic and necrotic processes suggest severe and prolonged visceral pain may be present in infants with NEC [50]. In addition, NEC is associated with an increase in painful interventions, up to 19 times a day, during 5 consecutive days [51]. Continuous morphine has been shown to adequately treat the pain associated with NEC in infants who require surgery [50]. However, a single-center study showed that pain assessment was performed in only $30-60 \%$ of NEC cases, while analgesia was used in only $52-76 \%$ of cases [51]. In a Delphi consensus study, inadequate pain management has been identified as a risk factor for developing chronic pain states in infants [52].

Although there are no studies that investigate the consequences of neonatal prolonged or chronic pain, followup studies indicate that pain in neonates in general may have long-term effects. Two recent studies looked at associations between perinatal factors, pain behavior, and pain-coping styles in adolescents who were born prematurely. In multivariate Cox regression analysis, NEC was an independent predictor for lower pain threshold (hazard ratio [HR] 1.47; 95\% CI 1.01-2.14) and pain tolerance (HR 1.63; 95\% CI 1.09-2.41) as measured with a cold pressor test [53]. In contrast, in the same cohort NEC was not associated with altered pain-coping styles [54].

\section{Inflammation}

Intestinal injury and barrier dysfunction permit the translocation of bacteria and inflammatory mediators to the systemic circulation, resulting in a systemic inflammatory response. The cytokines which are produced by this process may disrupt development of the vulnerable preterm brain [3]. Volpe [55] demonstrated that in the process of encephalopathy the premyelinating oligodendrocyte is very vulnerable to ischemia and inflammation. As the preoligodendrocyte plays an essential role in preterm brain development, damage to these cells may disrupt brain development. Indeed, in a multicenter study in infants with NEC, increased levels of pro-inflammatory cytokines were associated with poor growth and neurodevelopment [56]. Increased NEC severity (extension of injury to the intestine) was associated with an adverse neurocognitive outcome [57].

\section{Microbiota}

The proposed pathways for the direct effect of the microbiota on brain functioning and development are several. First of all, microbiota cause and may intensify systemic inflammation. Furthermore, dysbiosis may lead (via SCFA production) to decreased gut barrier and bloodbrain barrier functioning. Besides, microbiota may influence the brain by neurotransmitter (serotonin, melatonin, GABA, dopamine) production or incite gut cells to do so. Gut microbiota can elicit signals to the brain and vice versa via the vagal nerve system. SFCAs may have a direct effect on brain function and development $[3,58,59]$.

Therefore, gut dysbiosis in NEC has the potential to harm neurodevelopment not only via inflammation, but 
also may be a factor in itself causing abnormal neurodevelopment. Interestingly, the administration of probiotics may mitigate the effect of dysbiosis on brain development [21]. However, more research into this relation between microbiota and brain development in the preterm infant, within but also outside the clinical picture of NEC, is warranted. However, current knowledge of the gut microbiota allows an application in the diagnosis of NEC.

\section{Microbiota-Based Diagnosis of NEC}

Various markers for the early diagnosis of NEC have been investigated. These have been markers of inflammation (CRP, IL-6, and IL-8), markers of enterocyte damage (fatty acid binding protein), and markers of neutrophil migration and activity (calprotectin) [5].

As no single pathogen is known to cause NEC and changes in microbiota preceding NEC seem not to be very specific, microbiota analysis cannot be used for the early diagnosis or prediction of NEC. However, microbiotaderived metabolites which are produced by the interaction of microbiota with the host are known to play a role in the development of NEC. Within the field of metabolomics, fecal volatile organic compounds (VOCs) are being identified and increasingly studied. The changes in fecal VOCs preceding NEC may reflect a change in microbiota composition as well as a change in the metabolic activity of specific microbes.

To date, the potential of fecal VOCs for the detection of NEC in a preclinical phase has been explored in two studies. Garner et al. [60] compared fecal VOCs of 6 preterm infants with NEC with 7 controls in a preclinical phase study using gas chromatography-mass spectrometry (GC-MS). They found that the number of VOCs decreased with increasing age in control infants. Furthermore, four specific esters found in the control infants could not be detected in the infants who developed NEC [60].

In the second study, fecal VOCs were analyzed by means of an electronic nose (eNose) device. This technology allows for the comparison of fecal VOC profiles based on pattern recognition algorithms. It is user friendly, lower in cost and less time-consuming than chemical analytical techniques. de Meij et al. [61] demonstrated that fecal VOC profiles allowed discrimination between cases $(n=13)$ and controls $(n=14)$ up to 3 days prior to the clinical onset of NEC, with increasing accuracy closer to the day of diagnosis. Interestingly, fecal VOC profiles obtained from preterm infants who developed NEC also dif-

NEC, Gut Microbiota, and Brain

Development: Role of the Brain-Gut Axis fered from infants who developed late-onset sepsis (LOS). Presumably, both disorders harbor a unique microbial and metabolic shift preceding disease onset, allowing preclinical discrimination [61]. This is of interest to the clinician, as even at clinical onset of the disease, clinical differentiation of NEC and LOS can be difficult.

In a subsequent study, the potential of fecal VOCs in the early detection of LOS was studied. The analysis of fecal VOCs by eNose could discriminate separate sepsis cases $(n=36)$ from controls $(n=40)$ up to 3 days preceding disease onset [62]. Since the causative agent of LOS is often abundantly present in the days preceding disease onset and each bacterial species produces a unique VOC fingerprint, we suggest that fecal VOCs may allow for the preclinical detection of pathogens at the species level [33]. Another recent trial by Stewart et al. [63] partly underlines this hypothesis. When preterm infants with LOS were compared to controls by microbiota and metabolomics analysis, no distinct conclusion could be made about the infants with LOS. However, in the infants without sepsis, higher abundance of Bifidobacterium was found. The abundance of Bifidobacterium was related to metabolites which were associated with growth of these specific bacteria. However, multicenter studies including a large cohort of infants with sepsis with a wide variety of LOScausing pathogens and identification of species-specific VOCs are needed to confirm this hypothesis.

An important disadvantage of the eNose technique is that it does not allow for identification of untargeted, individual VOCs. If fecal VOC profiles allow for the differentiation between disease and control subjects, identification of discriminating VOCs and unravelling underlying pathophysiological processes is hampered. To date, there is no unifying hypothesis related to altered metabolism in NEC from published studies and the use of metabolomic techniques in understanding NEC pathogenesis is in its infancy. Integration of all omics platforms is needed to understand the pathophysiology of NEC.

\section{Conclusion}

NEC is a relatively frequent disease in preterm infants, resulting in high mortality and morbidity rates. Preterm infants who survive NEC are at increased risk of neurocognitive deficits. Although the exact etiology of NEC has not been elucidated, microbiota play a major role in its development by inciting a TLR4-mediated inflammatory cascade [41].

Neonatology 2019;115:423-431

DOI: $10.1159 / 000497420$ 
Microbiota are not only related to the development of NEC and inflammation, but also are suggested to have a direct effect on brain functioning and development in preterm infants. In this review, we have discussed the evidence from experimental studies on the relation between gut microbiota and brain development. Gut microbiota influence the brain by generation of neurotransmitters, SCFA production, immune signaling (inflammatory mediators like cytokines and immune cells), and direct afferent nerve communication (vagal nerve). Therefore, research into microbial metabolites arising from microbiota-host interaction may be of interest not only in the early diagnosis of NEC and LOS, but also by investigating the influence of microbiota on cerebral functioning and neurocognitive outcome.

Fecal VOC analysis is a novel field in metabolomics; VOCs reflect microbiota composition, function and microbiota-host interaction. VOC analysis can be per- formed by an electronic nose device, which is based on pattern recognition, is user friendly, low cost and less time consuming compared to GC-MS, which is based on the characterization of individual VOCs. Therefore, gut metabolome analysis by fecal VOC analysis using eNose technology may provide the opportunity to assess composition, function, and activity, and thus the role of microbiota in NEC, sepsis, and neurodevelopment. This may provide the opportunity to identify and develop protective strategies against NEC and impaired neurodevelopment in daily clinical practice, such as targeted, rational-based supplementation of pre- and probiotics.

\section{Disclosure Statement}

The authors declare that no conflicts of interest exist.

\section{References}

1 Neu J, Walker WA. Necrotizing enterocolitis. N Engl J Med. 2011 Jan;364(3):255-64.

2 Yee WH, Soraisham AS, Shah VS, Aziz K, Yoon W, Lee SK; Canadian Neonatal Network. Incidence and timing of presentation of necrotizing enterocolitis in preterm infants. Pediatrics. 2012 Feb;129(2):e298-304.

3 Hickey M, Georgieff M, Ramel S. Neurodevelopmental outcomes following necrotizing enterocolitis. Semin Fetal Neonatal Med. 2018 Dec;23(6):426-32.

4 Neu J. Necrotizing enterocolitis: the mystery goes on. Neonatology. 2014;106(4):289-95.

5 Niemarkt HJ, de Meij TG, van de Velde ME, van der Schee MP, van Goudoever JB, Kramer BW, et al. Necrotizing enterocolitis: a clinical review on diagnostic biomarkers and the role of the intestinal microbiota. Inflamm Bowel Dis. 2015 Feb;21(2):436-44.

6 Sharma R, Hudak ML. A clinical perspective of necrotizing enterocolitis: past, present, and future. Clin Perinatol. 2013 Mar;40(1):27-51.

7 Neu J, Pammi M. Necrotizing enterocolitis: the intestinal microbiome, metabolome and inflammatory mediators. Semin Fetal Neonatal Med. 2018 Dec;23(6):400-5.

8 Moschopoulos C, Kratimenos P, Koutroulis I, Shah BV, Mowes A, Bhandari V. The neurodevelopmental perspective of surgical necrotizing enterocolitis: the role of the gut-brain axis. Mediators Inflamm. 2018 Mar;2018: 7456857.

9 Collins SM, Surette M, Bercik P. The interplay between the intestinal microbiota and the brain. Nat Rev Microbiol. 2012 Nov;10(11): $735-42$.
10 Sudo N, Chida Y, Aiba Y, Sonoda J, Oyama N, $\mathrm{Yu} \mathrm{XN}$, et al. Postnatal microbial colonization programs the hypothalamic-pituitary-adrenal system for stress response in mice. J Physiol. 2004 Jul;558(Pt 1):263-75.

11 Douglas-Escobar M, Elliott E, Neu J. Effect of intestinal microbial ecology on the developing brain. JAMA Pediatr. 2013 Apr;167(4): 374-9.

12 Diaz Heijtz R. Fetal, neonatal, and infant microbiome: perturbations and subsequent effects on brain development and behavior. Semin Fetal Neonatal Med. 2016 Dec;21(6): 410-7.

13 Lyte M, Varcoe JJ, Bailey MT. Anxiogenic effect of subclinical bacterial infection in mice in the absence of overt immune activation. Physiol Behav. 1998 Aug;65(1):63-8.

14 Goehler LE, Gaykema RP, Opitz N, Reddaway $\mathrm{R}$, Badr N, Lyte M. Activation in vagal afferents and central autonomic pathways: early responses to intestinal infection with Campylobacter jejuni. Brain Behav Immun. 2005 Jul; 19(4):334-44.

15 Holzer P, Farzi A. Neuropeptides and the microbiota-gut-brain axis. Adv Exp Med Biol. 2014;817:195-219.

$16 \mathrm{Lu} \mathrm{J}$, Claud EC. Connection between gut microbiome and brain development in preterm infants. Dev Psychobiol. 2018 Nov; Epub ahead of print. https://doi.org/10.1002/dev.21806.

17 Lyte M. Probiotics function mechanistically as delivery vehicles for neuroactive compounds: microbial endocrinology in the design and use of probiotics. BioEssays. 2011 Aug;33(8):574-81.
18 Desbonnet L, Garrett L, Clarke G, Kiely B, Cryan JF, Dinan TG. Effects of the probiotic Bifidobacterium infantis in the maternal separation model of depression. Neuroscience. 2010 Nov; $170(4): 1179-88$

19 Ledochowski M, Widner B, Sperner-Unterweger B, Propst T, Vogel W, Fuchs D. Carbohydrate malabsorption syndromes and early signs of mental depression in females. Dig Dis Sci. 2000 Jul;45(7):1255-9.

20 Honda K, Takeda K. Regulatory mechanisms of immune responses to intestinal bacteria. Mucosal Immunol. 2009 May;2(3):187-96.

21 Keunen K, van Elburg RM, van Bel F, Benders MJ. Impact of nutrition on brain development and its neuroprotective implications following preterm birth. Pediatr Res. 2015 Jan;77(1-2):148-55.

22 Jilling T, Simon D, Lu J, Meng FJ, Li D, Schy $\mathrm{R}$, et al. The roles of bacteria and TLR4 in rat and murine models of necrotizing enterocolitis. J Immunol. 2006 Sep;177(5):3273-82.

23 Morowitz MJ, Poroyko V, Caplan M, Alverdy $\mathrm{J}$, Liu DC. Redefining the role of intestinal microbes in the pathogenesis of necrotizing enterocolitis. Pediatrics. 2010 Apr;125(4):77785.

24 Claud EC, Walker WA. Hypothesis: inappropriate colonization of the premature intestine can cause neonatal necrotizing enterocolitis. FASEB J. 2001 Jun;15(8):1398-403.

25 Jiménez E, Marín ML, Martín R, Odriozola $\mathrm{JM}$, Olivares M, Xaus J, et al. Is meconium from healthy newborns actually sterile? Res Microbiol. 2008 Apr;159(3):187-93. 
26 Patel RM, Denning PW. Intestinal microbiota and its relationship with necrotizing enterocolitis. Pediatr Res. 2015 Sep;78(3):232-8.

27 Aagaard K, Ma J, Antony KM, Ganu R, Petrosino J, Versalovic J. The placenta harbors a unique microbiome. Sci Transl Med. 2014; 6(237):65.

28 Rakoff-Nahoum S, Paglino J, Eslami-Varzaneh F, Edberg S, Medzhitov R. Recognition of commensal microflora by toll-like receptors is required for intestinal homeostasis. Cell. 2004 Jul;118(2):229-41.

29 Abreu MT. Toll-like receptor signalling in the intestinal epithelium: how bacterial recognition shapes intestinal function. Nat Rev Immunol. $2010 \mathrm{Feb} ; 10(2): 131-44$.

30 La Rosa PS, Warner BB, Zhou Y, Weinstock GM, Sodergren E, Hall-Moore CM, et al. Patterned progression of bacterial populations in the premature infant gut. Proc Natl Acad Sci USA. 2014 Aug;111(34):12522-7.

31 Penders J, Thijs C, Vink C, Stelma FF, Snijders B, Kummeling I, et al. Factors influencing the composition of the intestinal microbiota in early infancy. Pediatrics. 2006 Aug; 118(2):511-21.

32 Pammi M, Cope J, Tarr PI, Warner BB, Morrow $\mathrm{AL}$, Mai $\mathrm{V}$, et al. Intestinal dysbiosis in preterm infants preceding necrotizing enterocolitis: a systematic review and metaanalysis. Microbiome. 2017 Mar;5(1):31.

33 Berkhout DJ, Niemarkt HJ, de Boer NK, Benninga MA, de Meij TG. The potential of gut microbiota and fecal volatile organic compounds analysis as early diagnostic biomarker for necrotizing enterocolitis and sepsis in preterm infants. Expert Rev Gastroenterol Hepatol. 2018 May;12(5):457-70.

34 Morrow AL, Lagomarcino AJ, Schibler KR Taft DH, Yu Z, Wang B, et al. Early microbial and metabolomic signatures predict later onset of necrotizing enterocolitis in preterm infants. Microbiome. 2013 Apr;1(1):13.

35 Zhou Y, Shan G, Sodergren E, Weinstock G, Walker WA, Gregory KE. Longitudinal analysis of the premature infant intestinal microbiome prior to necrotizing enterocolitis: a case-control study. PLoS One. 2015 Mar 10(3):e0118632.

36 Warner BB, Deych E, Zhou Y, Hall-Moore C, Weinstock GM, Sodergren E, et al. Gut bacteria dysbiosis and necrotising enterocolitis in very low birthweight infants: a prospective case-control study. Lancet. 2016 May; 387(10031):1928-36.

37 Gregory KE, Samuel BS, Houghteling P, Shan G, Ausubel FM, Sadreyev RI, et al. Influence of maternal breast milk ingestion on acquisition of the intestinal microbiome in preterm infants. Microbiome. 2016 Dec;4(1):68.

38 Hackam DJ, Sodhi CP, Good M. New insights into necrotizing enterocolitis: from laboratory observation to personalized prevention and treatment. J Pediatr Surg. 2019 Mar; 54(3):398-404
39 Neu J, Pammi M. Pathogenesis of NEC: im pact of an altered intestinal microbiome. Semin Perinatol. $2017 \mathrm{Feb}$;41(1):29-35.

40 Nanthakumar N, Meng D, Goldstein AM, Zhu W, Lu L, Uauy R, et al. The mechanism of excessive intestinal inflammation in necrotizing enterocolitis: an immature innate immune response. PLoS One. 2011 Mar; 6(3):e17776.

41 Hackam D, Caplan M. Necrotizing enterocolitis: pathophysiology from a historical context. Semin Pediatr Surg. 2018 Feb;27(1):11-8.

42 Hackam DJ, Sodhi CP: Toll-like receptor-mediated intestinal inflammatory imbalance in the pathogenesis of necrotizing enterocolitis. Cell Mol Gastroenterol Hepatol. 2018;6(2): 229-238.e1. https://doi.org/10.1016/j.jcmgh. 2018.04.001.

43 Byndloss MX, Olsan EE, Rivera-Chávez F, Tiffany CR, Cevallos SA, Lokken KL, et al. Microbiota-activated PPAR- $\gamma$ signaling inhibits dysbiotic Enterobacteriaceae expansion. Science. 2017 Aug;357(6351):570-5.

44 Lin J. Too much short chain fatty acids cause neonatal necrotizing enterocolitis. Med $\mathrm{Hy}$ potheses. 2004;62(2):291-3.

45 Ge X, Pan J, Liu Y, Wang H, Zhou W, Wang $\mathrm{X}$. Intestinal crosstalk between microbiota and serotonin and its impact on gut motility. Curr Pharm Biotechnol. 2018;19(3):190-5.

46 Schulzke SM, Deshpande GC, Patole SK. Neurodevelopmental outcomes of very lowbirth-weight infants with necrotizing enterocolitis: a systematic review of observational studies. Arch Pediatr Adolesc Med. 2007 Jun; 161(6):583-90

47 Rees CM, Pierro A, Eaton S. Neurodevelopmental outcomes of neonates with medically and surgically treated necrotizing enterocolitis. Arch Dis Child Fetal Neonatal Ed. 2007 May;92(3):F193-8.

48 Dong Y, Speer CP. Late-onset neonatal sepsis: recent developments. Arch Dis Child Fetal Neonatal Ed. 2015 May;100(3):F257-63.

49 Martin CR, Dammann O, Allred EN, Patel S, O'Shea TM, Kuban KC, Leviton A: Neurodevelopment of extremely preterm infants who had necrotizing enterocolitis with or without late bacteremia. J Pediatr. 2010;157:751-756. e1.

50 Meesters NJ, van Dijk M, Knibbe CA, KeyzerDekker CM, Tibboel D, Simons SH. Infants operated on for necrotizing enterocolitis: towards evidence-based pain guidelines. Neonatology. 2016;110(3):190-7.

51 Gibbins S, Maddalena P, Moulsdale W, Gar$\operatorname{rard}$ F, jan Mohamed T, Nichols A, et al. Pain assessment and pharmacologic management for infants with NEC: a retrospective chart audit. Neonatal Netw. 2006 Sep-Oct;25(5):33945.
52 van Ganzewinkel CJ, Anand KJ, Kramer BW, Andriessen P. Chronic pain in the newborn: toward a definition. Clin J Pain. 2014 Nov; 30(11):970-7.

53 van Ganzewinkel CJ, Been JV, Verbeek I, van der Loo TB, van der Pal SM, Kramer BW, et al Pain threshold, tolerance and intensity in adolescents born very preterm or with low birth weight. Early Hum Dev. 2017 Jul;110:31-8.

54 van Ganzewinkel CJ, Been JV, Dieleman JP, Katgert T, Boelen-van der Loo T, van der Pal SM, et al. Pain coping strategies: neonatal intensive care unit survivors in adolescence. Early Hum Dev. 2016 Dec;103:27-32.

55 Volpe JJ. Systemic inflammation, oligodendroglial maturation, and the encephalopathy of prematurity. Ann Neurol. 2011 Oct;70(4): 525-9.

56 Lodha A, Asztalos E, Moore AM. Cytokine levels in neonatal necrotizing enterocolitis and long-term growth and neurodevelopment. Acta Paediatr. 2010 Mar;99(3):338-43.

57 Shah TA, Meinzen-Derr J, Gratton T, Steichen J, Donovan EF, Yolton K, et al. Hospital and neurodevelopmental outcomes of extremely low-birth-weight infants with necrotizing enterocolitis and spontaneous intestinal perforation. J Perinatol. 2012 Jul;32(7): $552-8$.

58 Diaz Heijtz R, Wang S, Anuar F, Qian Y, Björkholm B, Samuelsson A, et al. Normal gut microbiota modulates brain development and behavior. Proc Natl Acad Sci USA. 2011 Feb;108(7):3047-52.

$59 \mathrm{Lu} \mathrm{J,} \mathrm{Lu} \mathrm{L,} \mathrm{Yu} \mathrm{Y,} \mathrm{Cluette-Brown} \mathrm{J,} \mathrm{Martin} \mathrm{CR,}$ Claud EC. Effects of intestinal microbiota on brain development in humanized gnotobiotic mice. Sci Rep. 2018 Apr;8(1):5443.

60 Garner CE, Ewer AK, Elasouad K, Power F, Greenwood R, Ratcliffe NM, et al. Analysis of faecal volatile organic compounds in preterm infants who develop necrotising enterocolitis: a pilot study. J Pediatr Gastroenterol Nutr. 2009 Nov;49(5):559-65.

61 de Meij TG, van der Schee MP, Berkhout DJ, van de Velde ME, Jansen AE, Kramer BW, van Weissenbruch MM, van Kaam AH, Andriessen P, van Goudoever JB, Niemarkt HJ, de Boer NK: Early detection of necrotizing enterocolitis by fecal volatile organic compounds analysis. J Pediatr. 2015 Sep;167(3): 562-7.e1.

62 Berkhout DJ, Niemarkt HJ, Buijck M, van Weissenbruch MM, Brinkman P, Benninga $\mathrm{MA}$, et al. Detection of sepsis in preterm infants by fecal volatile organic compounds analysis: a proof of principle study. J Pediatr Gastroenterol Nutr. 2017 Sep;65(3):e47-52.

63 Stewart CJ, Embleton ND, Marrs EC, Smith DP, Fofanova T, Nelson A, et al. Longitudinal development of the gut microbiome and metabolome in preterm neonates with late onset sepsis and healthy controls. Microbiome. 2017 Jul;5(1):75
NEC, Gut Microbiota, and Brain

Development: Role of the Brain-Gut Axis
Neonatology 2019;115:423-431

DOI: $10.1159 / 000497420$ 